\title{
A Comparative Study of Conventional and Modern Phase Coded Radar Transmitters
}

\author{
Mohamed A. A. Abdel-Rahman \\ Electrical Engineering Department, Faculty of Engineering, Al-Baha University, Al-Baha, Saudi Arabia
}

\begin{abstract}
The main objective of this paper is to present the most important techniques applied to pulse radar transmitters such as a delay-line-type modulator (DLM) for conventional radars and a binary phase modulation for modern ones. A pulse forming network (PFN), comprising of a delay line with LC-sections, charging chock, blocking diode and silicon-controlled rectifier (SCR), is investigated. The pre-trigger signal with $2-\mathrm{kHz}$ pulse repetition frequency is responsible for beginning of the transmission period. The duration of transmitted pulse, generated by DLM, depends mainly on the number of LC-sections and their element values. The formed pulse feeds the radiofrequency (RF) oscillator (magnetron) as a high bias voltage to give the RF carrier. A 7-bit code generator is designed to control the phase of carrier signal using the ring modulator circuit aiming to minimizing the jamming signals effect and achieving security for the communication systems. Furthermore, the phase coding modulation facilitates the design and implementation of pulse compression circuit, digital correlator in our work, for modern radar receivers. This improves the resolving power in range and angle, signal to noise ratio (SNR) of the detected targets and the associated probability of detection.
\end{abstract}

Keywords: Ring Modulator; Pulse Compression; Signal to Noise Ratio, SNR; Pulse Forming Network; Binary Phasecoded Signal.

\section{INTRODUCTION}

Radio frequency energy in radar is transmitted in short pulses with time durations that may vary from 1 to $50 \mu \mathrm{s}$ or more. A special modulator is needed to produce this pulse of high voltage. The hydrogen thyratron modulator is the most common radar modulator. It employs a pulse forming network (PFN) [1-4], that is charged up slowly to a high value of voltage. The network is discharged rapidly through a pulse transformer by the thyratron tube to develop an output pulse, The shape and duration of the pulse are determined by the electrical characteristics of the pulse-forming network and of the pulse transformer. The problem of using such type of modulator is its inability to cope with the jamming signals which cause difficulty for the radar operator to detect or track the targets. Therefore the digital modulation becomes very powerful for best detection and accurate tracking to air flying targets especially with active jamming situations. This paper is organized as follows: Section II deals with the delay-linetype modulator for conventional radar transmitter, while the generation of phase-coded signal using ring modulator associated with correlation codes is dealt with in Section III. Section IV deals with maximal length sequences; Section V discusses simulation results; and Section VI presents concluding remarks.

\section{CONVENTIONAL RADAR TRANSMITTER}

The final stage of the conventional radar transmitter is the RF power oscillator (magnetron). For exciting such magnetron at the proper frequency and at the proper power level, the modulator must be designed for supplying high power pulses to it. The schematic diagram of the delayline-type modulator (DLM) is depicted in Fig. 1. Starting with the analysis of the RLC circuit fed by the DC voltage $V_{\text {inp }}$, the transfer function [5] becomes,

$$
\frac{V_{c}}{V_{\text {inp }}}=\frac{1 / L C}{s^{2}+(R / L) s+1 / L C}
$$

There are two roots of the characteristic equation which are

$$
s_{1,2}=-\frac{R}{2 L} \pm \sqrt{\left(\frac{R}{2 L}\right)^{2}-\frac{1}{L C}} .
$$

We consider the under-damped case in our work, $(R / 2 L)^{2} \prec(1 / L C)$. The step response in such case, as a general solution for $t \geq 0$, becomes:

$$
V_{c}(t)=1+\left(A_{1} \cos \left(\omega_{d} t\right)+A_{2} \sin \left(\omega_{d} t\right)\right) e^{-\alpha t} .
$$

For the initial conditions, $V_{c}(0)=0$ and $d V_{c}(t) /\left.d t\right|_{t=0}=0$, the constants $A_{1}=-1, A_{2}=-\alpha / \omega_{d}$ and the step input $V_{i n p}=1 \mathrm{~V}$. Consequently, the step response in Eq. (3) can be represented by

$$
V_{c}(t)=1+K e^{-\alpha t} \cos \left(\omega_{d} t+\phi\right)
$$

where $\alpha=(R / 2 L)=\xi \omega_{o}$ is the damping factor,

$$
\begin{aligned}
& \omega_{o}=(1 / \sqrt{L C}) \text { is the natural frequency, } \\
& \omega_{d}=\omega_{o} \sqrt{1-\xi^{2}} \text { is the damped frequency, }
\end{aligned}
$$

and $\xi=(R / 2) \sqrt{C / L}$ is the damping coefficient.

In Eq. (4), the term $K e^{-\alpha t} \cos \left(\omega_{d} t+\phi\right)$ represents the natural response and the unity represents the particular solution. 

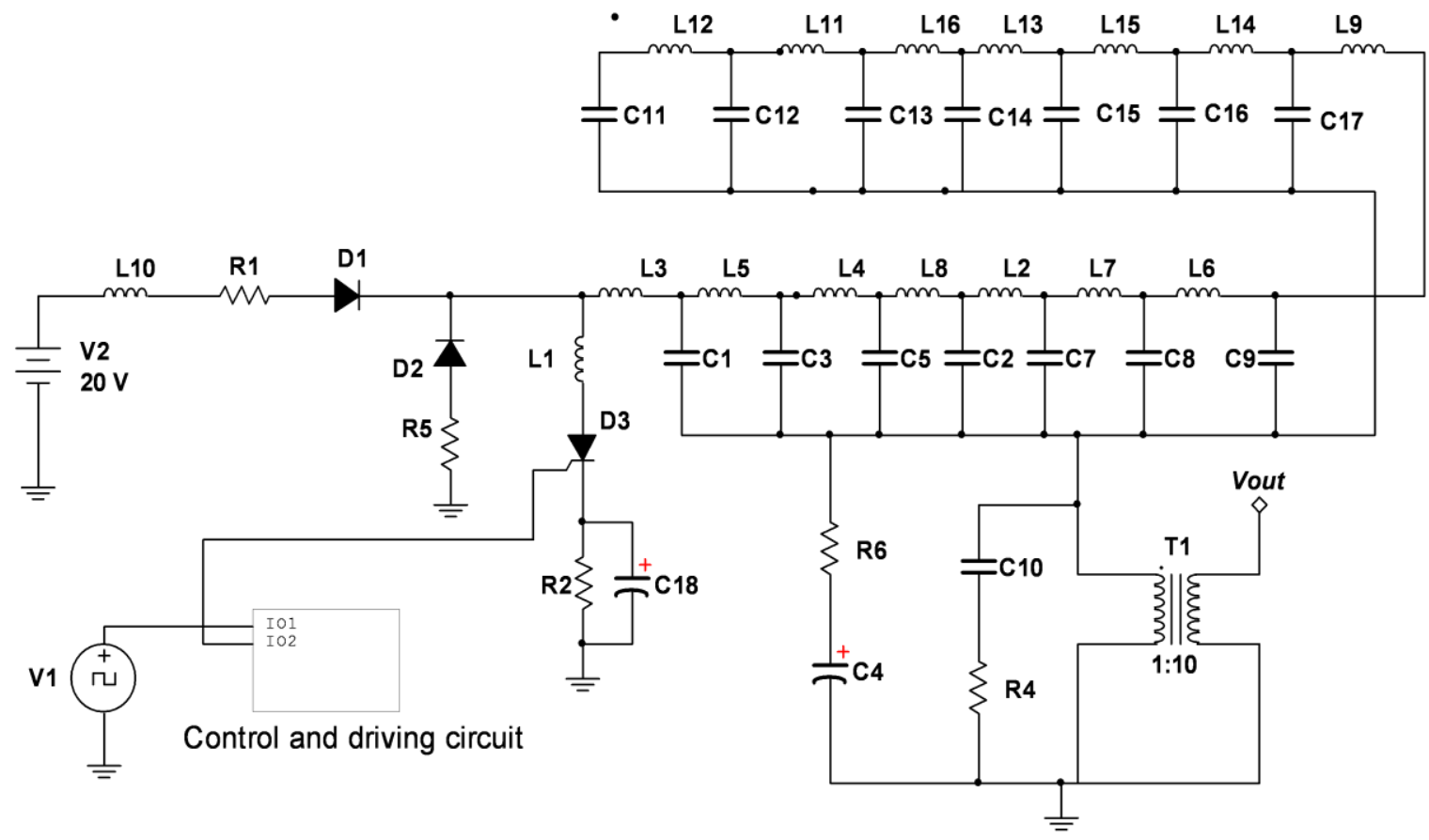

Fig. 1 Schematic diagram of a delay-line-type modulator

Consider the initially quiescent state (both the initial The first peak, computed from Eq. (7) with $n=1$, is inductance current and the initial capacitor voltage are zeros).

$$
\begin{aligned}
& \phi=-\tan ^{-1}\left(\alpha / \omega_{d}\right)=-\tan ^{-1}\left(\xi / \sqrt{1-\xi^{2}}\right) \\
& K=-(1 / \cos \phi)=-1 / \sqrt{1-\xi^{2}}
\end{aligned}
$$

The maxima and minima occur alternately when

$$
\tan \left(\omega_{d} t+\phi\right)=-\alpha / \omega_{d}, \omega_{d} t=n \pi, n=0,1,2,3 \ldots .
$$

From the above, the maximum and minimum values of

$V_{c}(t)$ are given by $\left.V_{C P K}(t)\right|_{t=\frac{n \pi}{\omega_{d}}}=1-(-1)^{n} e^{\frac{-n \pi \xi}{\sqrt{1-\xi^{2}}}}$

(odd values of $n$ give the maxima). The voltage step response of the RLC is computed and illustrated as in Fig. 2.

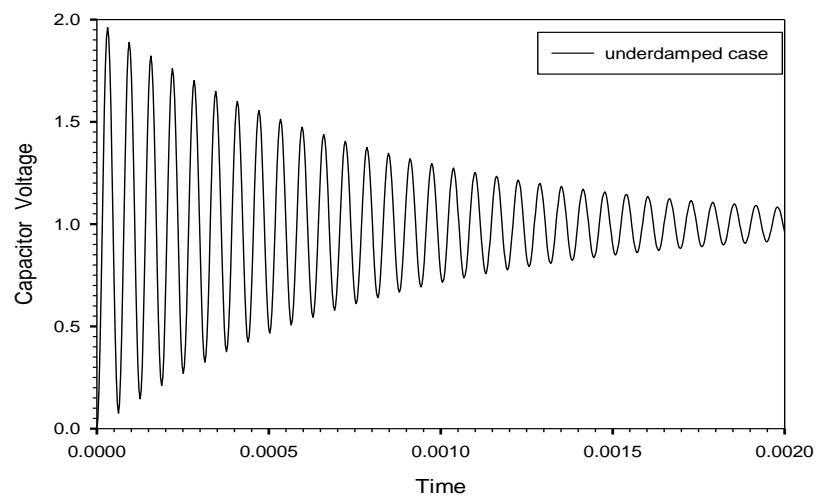
$1.96 V_{\text {inp }}$ at $t=\pi / \omega_{d}=31.4 \mu \mathrm{s}$ which approximately equals to twice its step input value as shown in Fig. 2.

The charging diode keeps such voltage value on the capacitor until the silicon-controlled rectifier (SCR) becomes energized by the gate signal. The parameters of the modulator in our analysis that affect the design can be summarized as: pulse width $\delta=14 \mu s, P R F=2 \mathrm{kHz}$ and the duty ratio, $D=0.028$.

The operation of the (DLM), shown in Fig. 1, is as follow: An artificial transmission line, used as PFN, is charged initially to a first peak voltage value $V_{C P K 1}$. At this instant and using $R_{L}=R_{C}=\sqrt{L / C}=100 \Omega$, the $\mathbf{S C R}$ is conducting one half of the voltage stored on the line will immediately appear across $R_{L}$ leaving the voltage across the input terminals of the line reduced to $V_{C P K 1} / 2$. This is equivalent to introducing a negative voltage of amplitude $V_{C P K 1} / 2$ to the input terminals. This wavefront will travel down the line at the propagation velocity to the open end, leaving the line charged with $V_{C P K 1} / 2$. The reflected wavefront with $\Gamma_{L}=1$ becomes $-V_{C P K 1} / 2$, and returns to the input end canceling the remaining voltage on the line. The generated pulse has a width $\delta=2 n \sqrt{L C}=14 \mu \mathrm{s}$.

Fig. 2 Voltage step response of KLC' circuit for $f_{o}=15.9 \mathrm{kHz}$ with $\mathrm{L}_{\mathrm{ch}}=20 \mathrm{mH}, \mathrm{C}=5 \mathrm{nF}$, and $\mathrm{R}=50 \Omega$. 


\section{MODERN TECHNIQUES OF RADAR TRANSMITTER}

Narrow pulse systems require large peak power ( $>1 \mathrm{MW}$ ) for long range operation and so special precautions must be taken to minimize the problems of ionization and arcing within the waveguide for radar systems. This makes it advantageous to generate a transmitted waveform that decouples the range resolution from the duration of the pulse. To cope with these precautions in modern radars, the pulse width, $\tau$, should be widened to alleviate such problems. This causes the fixed-frequency continuouswave $(\mathrm{CW})$ radar, in Section II, to be unable to resolve range,

$$
\delta R=c /(2 \Delta f), \text { and } \Delta f=1 / \tau .
$$

Frequency and phase modulation of the carrier [2-4, 6-8] are the most common techniques used to broaden this spectrum. Solutions involve lengthening the pulse to achieve large radiated energy, while still maintaining the wide bandwidth for good range-resolution $[6,9,10,11]$. The received signal can then be processed using a matched filter that compresses the long pulse to a duration $1 / \Delta \mathrm{f}$. The time-bandwidth product $\Delta \mathrm{f} . \tau$ of the uncompressed pulse is used as a figure of merit for such "pulse compression" systems. The phase-coded waveforms differ from FM waveforms in that the pulse is subdivided into a number of subpulses. The subpulses are of equal duration, and each has a particular phase which is selected in accordance with a given code sequence. The most widely used phase-coded waveform employs two phases and is called binary, or biphase, coding. Signal alternates between $o^{\circ}$ and $180^{\circ}$. Since the transmitted frequency is not usually a multiple of the reciprocal of the subpulse width, the coded signal is generally discontinuous at the phase reversal points. The functional block diagram, shown in Fig. 3, illustrates the modern radar receiver including the code generator and encoder circuits which we are interested in our work.

\section{MAXIMAL LENGTH SEQUENCES}

Various pseudo random codes are generated using linear feedback shift register (LFSR). The generator polynomial governs all the characteristics of the generator. For a given generator polynomial, there are two ways [12] of implementing LFSR known as Galois and Fibonacci feedback generators. Careful inspection reveals that the order of the Galois weights is opposite that of the Fibonacci weights. The Galois form is generally faster than the Fibonacci in hardware due to the reduced number of logic gates in feedback loop, thus making it the favored form. Shift register sequences having the maximum possible period for an $r$-stage shift register are called maximal length sequences or m-sequences. A primitive generator polynomial $[1,13]$ always yield an $m$ sequence. The maximum period of an $r$-stage shift register can be proven to be $2^{r}-1$. The $m$-sequences have three important properties, i.e., balance property, run-length property and shift-and-add property [13-17]. The total number $M$ of maximum-length sequences that may be obtained from an $n$-stage generator is

$$
M=(N / n) \Pi\left(1-\left(1 / p_{i}\right)\right),
$$

where $p_{i}$ are the prime factors of $N$. The m-sequences have good autocorrelation property and are being used in many applications. This is attributed to the first and third properties.In our research, the designed circuit is based on Galois feedback generator. To generate a PRN sequence, one must initially load the shift register with any initial binary number except zero. Table 1, shown below [4], includes feedback configurations needed to generate maximal length sequences with lengths up to 1023. The sequence with length 1023 corresponds to 10 stages and modulo- 2 sum of stages 10 and 7 is fed back to the input. The table contains only one feedback configuration for each code length.

Table 1 Maximal length sequences

\begin{tabular}{|c|c|c|c||}
\hline $\begin{array}{c}\text { Number } \\
\text { of } \\
\text { stages } \\
n\end{array}$ & $\begin{array}{c}\text { Length of } \\
\text { maximal } \\
\text { sequence, } \\
\mathrm{N}\end{array}$ & $\begin{array}{c}\text { Number of } \\
\text { maximal } \\
\text { sequences }, \\
\mathrm{M}\end{array}$ & $\begin{array}{c}\text { Feedback } \\
\text { stage } \\
\text { connections }\end{array}$ \\
\hline \hline 2 & 3 & 1 & 2,1 \\
\hline 3 & 7 & 2 & 3,2 \\
\hline 4 & 15 & 2 & 4,3 \\
\hline 5 & 31 & 6 & 5,3 \\
\hline 6 & 63 & 6 & 6,5 \\
\hline 7 & 127 & 18 & 7,6 \\
\hline 8 & 255 & 16 & $8,6,5,4$ \\
\hline 9 & 511 & 48 & 9,5 \\
\hline 10 & 1023 & 60 & 10,7 \\
\hline
\end{tabular}

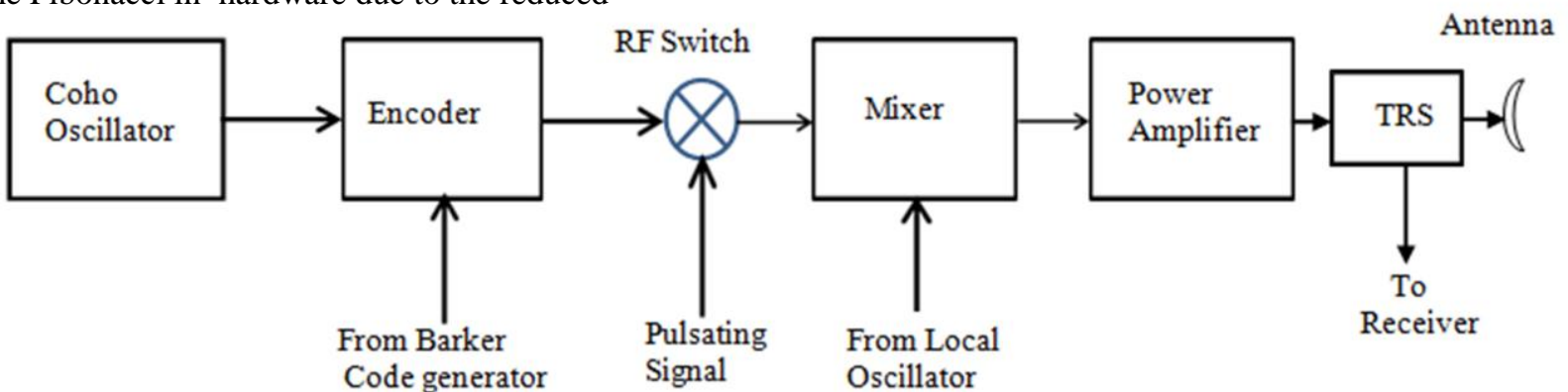

Fig. 3 Functional diagram of radar transmitter with binary phase coded signal 


\section{SIMULATION RESULTS}

For the purpose of simulation we have used DC voltage source of value $V_{\text {inp }}=20 \mathrm{~V}$ instead of $14 \mathrm{kV}$ in reality as well as the hydrogen thyratron is replaced by silicon controlled rectifier SCR. Figure 1 shows the schematic diagram of delay line type modulator [2-4] for the pulse radar with fixed carrier $f_{c}=2 \mathrm{MHz}$ and pulse repletion frequency (PRF) $f_{r}=2 \mathrm{kHz}$. The pulse duration, $\tau=14 \mu \mathrm{s}$, represents the transmission period and generated by 14 stages of $\mathrm{LC}$ sections. The reception period equals to $T_{r}-\tau=486 \mu \mathrm{s}$. The pulse transformer is capable to provide different voltage levels to the load, to perform dc isolation between the pulse forming network (PFN) and the load. Also to match the impedance level in order to transfer maximum power from PFN to the load (magnetron). The voltage waveforms at the anode of SCR and its gate are clarified in Fig. 4 to shed the light on the synchronization between them. A code generator of a 7-bit sequence [6-8] based on the linear feedback shift register (LFSR) [13,16-18] is clarified in Fig. 5. It consists of 3FFs, TTL-IC 7474, XOR and starting-up circuit.
All of the register elements share a common clock input of $1 \mathrm{MHz}$ with time period $1 \mu \mathrm{s}$. The initial loading, seed value, of the three-FFs is $\left(\begin{array}{lll}0 & 1 & 1\end{array}\right)$. In addition, the ring modulator $[14,20]$, depicted in Fig. 6 , to generate a binary phase coded signal as illustrated in Fig. 7. It has two input signals, one from the code generator and the other from RF carrier source. A subcircuit is designed to convert the unipolar code into its equivalent polar form to control the switching periods of the signal diodes. During a positive half voltage period, the RF carrier signal passes from secondary of the left transformer to the primary of the transformer at the right with the same phase and the side diodes become in reverse. Similarly during a negative half voltage period, the carrier passes through the side diodes and the top-bottom diodes become in reverse causing polarity inversion between the two transformers. This action is much like that of a DPDT (double-pole, doublethrow) switch wired for reversing connections. The signal diodes used having part number 1N4148 and characterized by high speed switching with max. 4 ns. The Matlab program has been performed to compute the autocorrelation function $\mathrm{AC}(\mathrm{k})$ for Barker code (1110010) $[2-4,6,20]$ as shown in Fig. 8. The Maximum sidelobe reduction is $16.9 \mathrm{~dB}$.
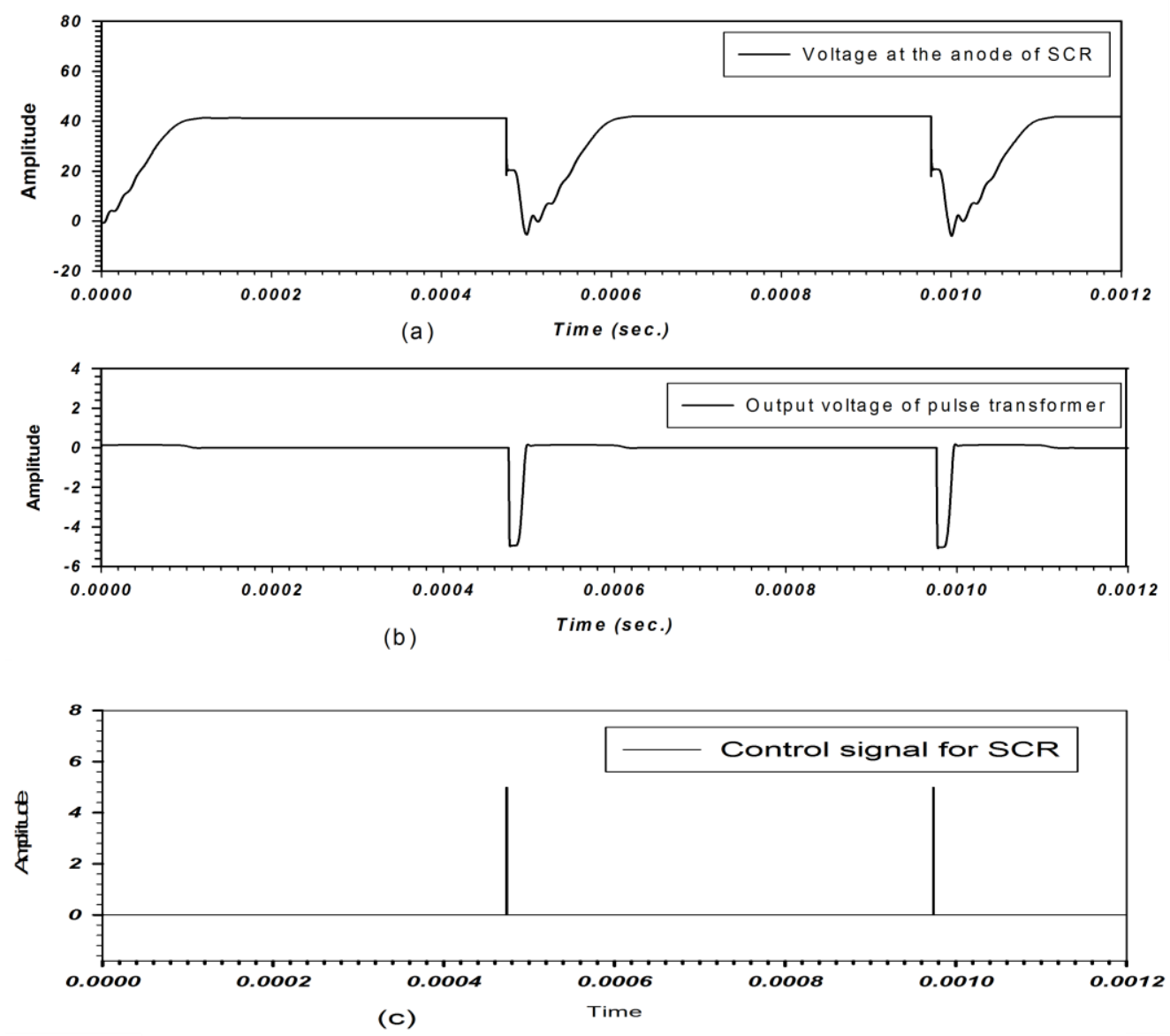

Fig. 4 Voltage waveforms of the delay line modulator

a) at the anode of the SCR.

b) at the output of pulse transformer

c) at the gate of the SCR. 


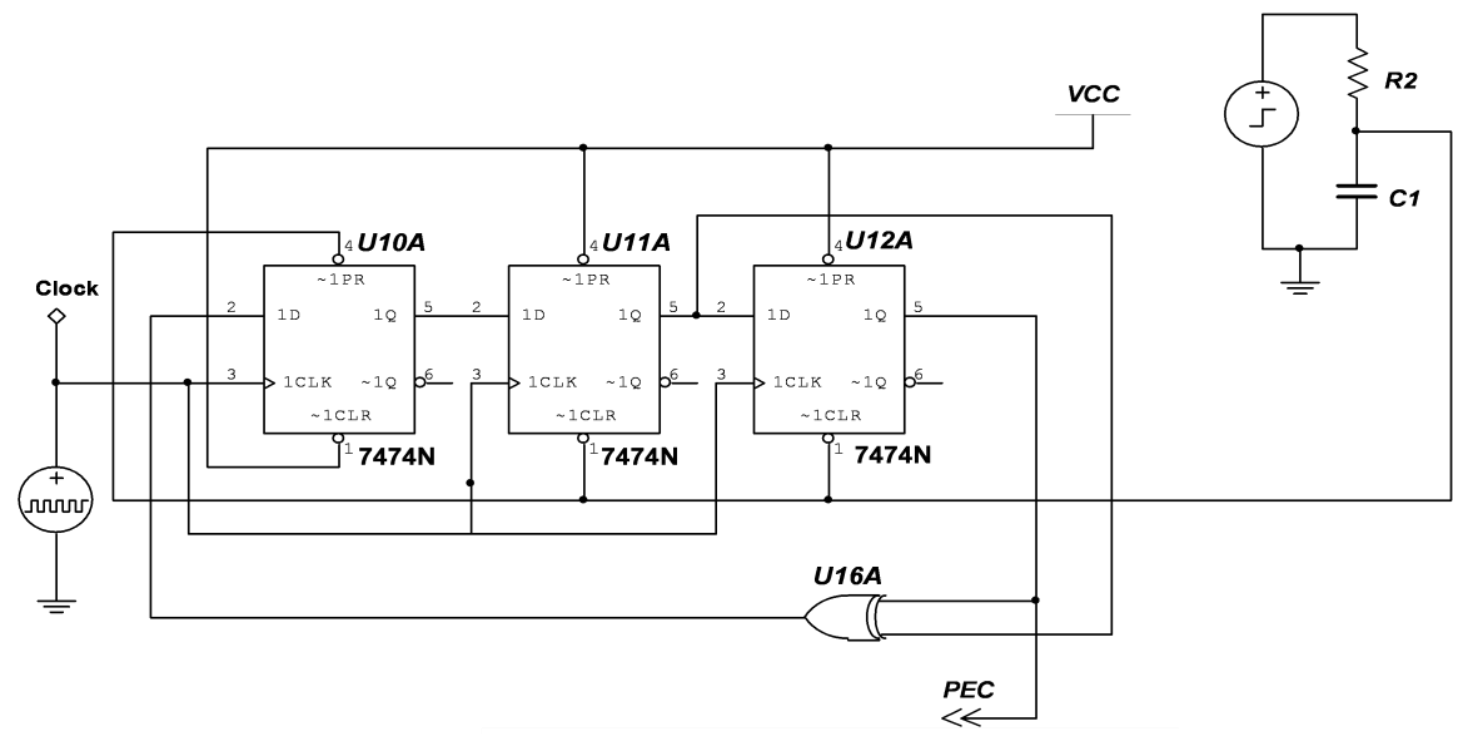

Fig. 5 7-bit Barker code generator

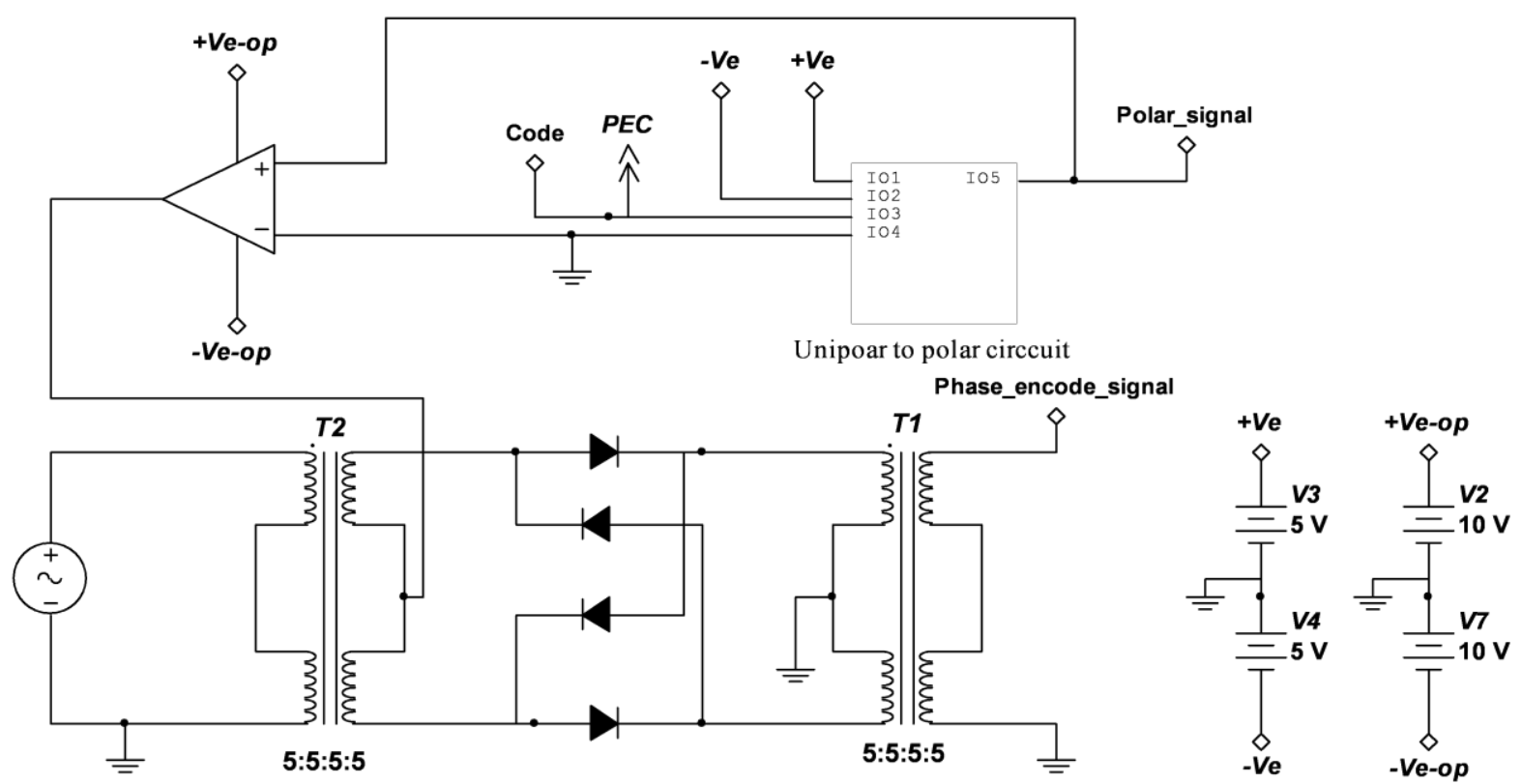

Fig. 6 Phase encoding circuit using 7 bit Barker code

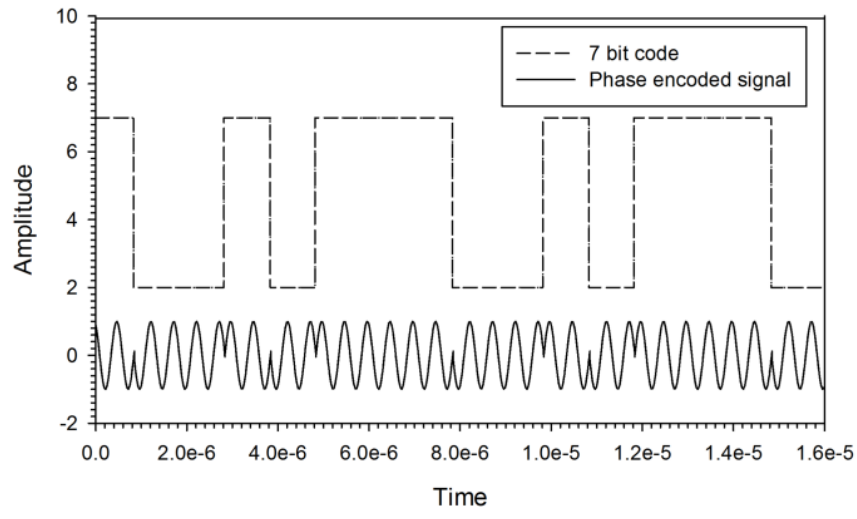

Fig. 7 Voltage waveform at the output of ring modulator for 7-bit code generated by 3 -stage shift register.

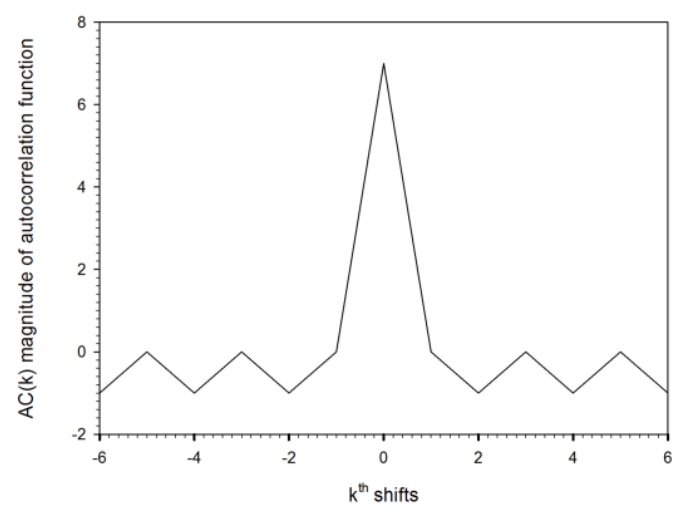

Fig. 8 Auto correlation of 7 bit Barker code (1110010) generated by LFSR. 


\section{CONCLUSION}

In this work, we present a comprehensive study of conventional and phase coded radar transmitters. We focused on the DLM which is used to energize a variety of high-power microwave devices such as magnetron and klystron. The generation of RF biphase coded signals using correlation codes, PN sequences, with variable lengths and their applications in modern radar systems are addressed. Specifically in the design of Barker code generator for transmitter and pulse compression circuit for receiver. This increases the immunity of radars to the interfering signals and enhances their performance and resolution. The simulated results confirm a good agreement with the computed ones. Along the same procedures, the other sequences mentioned in Table 1 can be generated. Such sequences have other enormous applications like Direct Sequence Spread Spectrum (DSSS), Built in Self Test (BIST) and DecryptionEncryption System (DES) error detection. A combined two channels I, Q (sine-cosine) to generate encoded signal with phase variation each $90^{\circ}$ will be studied in the next publication to alleviate the blind phase problem of the detected targets.

\section{REFERENCES}

[1] Fred E. Nathanson, J. Patrick Reill, and Marvin N. Cohen, Radar Design Principles: Signal Processing and the Environment, 2/Ed., Scitech Publishing Inc., Mendham, New Jersey, 1991.

[2] Merrill Skolnik, Introduction to Radar Systems, 3/Ed., McGraw Hill, Boston, 2001.

[3] William L. Melvin, James A. Scheer, Principles of Modern Radar Advanced Techniques, Scitech Publishing, Edison, NJ. , 2013.

[4] Merrill I. Skolnik, Radar Handbook, 3/Ed., McGraw Hill, 2007.

[5] Charles K. Alexander and Matthew N. Sadiku, Fundamentals of Electric Circuits, $4^{\text {th }}$ Ed., pages, 331-332, McGraw-Hill, 2009.

[6] Bassem R. Mahafza, Radar System Analysis \& Design Using MATLAB, CRC Press, 2005.

[7] Nedav Levanon and E. Mozeson, Radar Signals, Wiley, 2004.

[8] Chandan Singh D. Rawat and Anuja D. Sarate, "High Resolution Low Power Radar Pulse Compression Techniques", International Journal of Advanced Research in Electrical, Electronics and Instrumentation Engineering, Vol. 3, Issue 4, April 2014.

[9] Falih H. Ahmad, Design of a High-Resolution, Coded, Portable Radar System, Technical Report, U.S. Army Corps of Engineers, 1996

[10] G. E. Coxson and J. Russo, "Efficient Exhaustive Search for Optimal-Peak-Sidelobe Binary Codes," 2004 National Radar Conference, Philadelphia, PA, USA, May, 2004

[11] August W. Rihaczek, and Steven J. Hershkowitz, Radar Resolution and Complex-Image Analysis, Artech House Radar Library, 1996.

[12] Mark Goresky and Andrew Klapper, "Fibonacci and Galois Representations of Feedback-with-Carry Shift registers," IEEE Transactions on Information Theory, Vol.:48, Issue: 11, Nov., 2002

[13] Priyanka Shrivastava, Prashant Purohit, Pushpraj Singh Tanwar, and Harishanker Shrivastava, "Concepts of Primitive Polynomial and Galois Field in Designing More Randomize PN Sequence Generators for Maximum Fault Coverage in Modern VLSI Testing," International Journal of Innovative Science and Modern Engineering (IJISME) ISSN: 2319-6386, Vol. 2, Issue-10, September, 2014.

[14] B. P. Lathi and Zhi Ding, Modern Digital and Analog Communication Systems, The Oxford Series in Electrical and Computer Engineering, 4/Ed. , 2009.

[15] Mark A. Richards, Fundamentals of Radar Signal Processing," 2/Ed. , McGraw Hill, 2005.
[16] A. Ahmad, and A. Al-Maashri "Investigating Some Special Sequence Length Generated through an External Exclusive-NOR Type LFSRs," International Journal of Electrical and Computer Eng., Vol 34(1), 270-280, 2008.

[17] A. Ahmad, and A. M. Elabdalla. "An Efficient Method to Determine Linear Feedback Connections in Shift Registers that Generate Maximal Length Pseudo-random up and down Binary Sequences," Computer \& Electrical Engineering (Elsevier), Vol. 23(1), 33-39, 1997.

[18] D. K. Barton, Modern Radar System Analysis, Boston, MA: Artech House, 1988

[19] A. NagaJyothi and K. Raja Rajeswari, "Cross-correla-tion of Barker Code and Long Binary Signals." International Journal of Engineering Science 3.12, 8348-8356, 2011.

[20] Atul P. Godse, Uday Bakshi, Analog Communication, Technical Publications; 2/Ed., Jan., 2013.

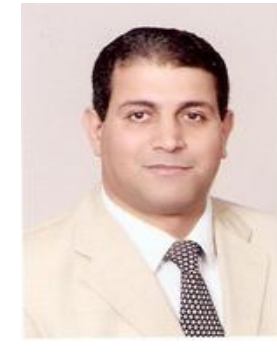

\section{BIOGRAPHY}

Mohamed A. A. Abdel-Rahman was born in Mansoura, Egypt, on February, 1955. He received the Bachelor of Science degree in electrical engineering from the Military Technical College, Cairo, Egypt in 1979 and the Master of Science degree from the Faculty of Engineering, University of Alexandria, Alexandria, Egypt in 1990. He received his Ph. D. degree from Virginia Polytechnic Institute and State University, USA in 1997 . He is presently working as an Assistant Professor at Faculty of Engineering of Al-Baha University, Saudi Arabia. His research and teaching interest areas include radar signal processing, digital power electronics and phased array radar. 